\title{
Películas fluorescentes azules basadas en derivados de poli-2,7-fluorenofenilideno
}

\author{
R. MALLAVIA A*, D.MARTINEZ-PERÉZ ${ }^{\text {A }}$, B.F. CHMELKA ${ }^{\text {B }}$, G.C. BAZAN ${ }^{\text {B }}$ \\ ${ }^{a}$ Instituto de Biología Molecular y Celular, Universidad Miguel Hernández, Elche 03202 Alicante (Spain). \\ ${ }^{\mathrm{b}}$ Departments of Chemical Engineering and Chemistry, University of California, Santa Barbara, CA 93106 (USA).
}

\begin{abstract}
La cuaternización del poli-(9,9-bis(6'-bromohexilo)fluorenofenileno) por tratamiento de trimetilamina gaseosa se emplea para obtener una sal de amonio del copolímero precursor soluble en agua. El copolímero neutro contiene unidades alternantes repetitivas de fluoreno/ fenilideno obtenidas mediante una acoplamiento de Suzuki con Pd (II). Los polímeros se caracterizaron por cromatografía de permeación (GPC), espectroscopia RMN de ${ }^{1} \mathrm{H}$ y ${ }^{13} \mathrm{C}$, espectroscopia IR transformada de Fourier y análisis termogravimétrico (ATG). El polímero neutro mantiene la estabilidad hasta $\operatorname{los} 300^{\circ} \mathrm{C}$, mientras el derivado catiónico comienza a descomponer a $120^{\circ} \mathrm{C}$, con una progresiva perdida de masa hasta los $290^{\circ} \mathrm{C}$. Las propiedades ópticas de los polímeros se estudiaron en disolución y en películas mediante espectroscopias UV/VIS y de fluorescencia. Las películas delgadas de sílice mesoporosa se prepararon como materiales receptores de los copolímeros fluorescentes.

Palabras clave: Polímeros conjugados, solubles en agua, Pd(II), silice mesoporosa.

Blue fluorescent films based on poly-2,7-fluorene-phenylene derivatives.

Quaternization of poly-(9,9-bis(6'-bromohexyl)fluorenephenylene by treatment with trimethylamine gas was used to obtain a water soluble ammonium salt copolymer. The neutral copolymer containing fluorene/ phenylene alternating repeating units was obtained by a palladiumcatalyzed Suzuki coupling reaction. This strategy could be applied to prepare water soluble conjugated polymers with the ability to change the charge functionality. The polymers were characterized by gel permeation chromatography (GPC), ${ }^{1} \mathrm{H}$ and ${ }^{13} \mathrm{C}$ NMR spectroscopy, FT-IR spectroscopy, and thermogravimetric analysis (TGA). The neutral polymer was stable to over $300^{\circ} \mathrm{C}$, while the cationic polymer begins to degrade at $120^{\circ} \mathrm{C}$ with a progressive loss of mass at $290^{\circ} \mathrm{C}$. The optical properties of the polymers were investigated in solution and solid phases by UV/VIS and fluorescent spectroscopy. Mesoporous silica thin films were prepared as a host matrix for the fluorescent copolymers.
\end{abstract}

Keywords: Conjugated polymers, water soluble, Pd (II), mesoporous silica.

\section{INTRODUCCIÓN}

Los polímeros conjugados fluorescentes con cadena principal de 2,7-fluoreno están siendo objeto de atención como componentes moleculares en la preparación de dispositivos optoelectrónicos (1-6). La gran versatibilidad en la preparación y modificación estructural los hace candidatos excelentes para estudiar el efecto que producen en las propiedades del material. Sin embargo, hay que tener presente que se modifican o cambian las propiedades de los polímeros conjugados dependiendo de la capacidad de dispersión de cada lote de fabricación (7). La obtención de polímeros conjugados cargados mejoraría la aplicabilidad de las sondas fluorescentes en análisis directos de moléculas biológicas $(7,8)$ o en la incorporación de composiciones iniciales de matrices inorgánicas $(9,10)$.

La incorporación de sustratos orgánicos con propiedades específicas se realiza en matrices de sílice ordenadas, estables térmicamente, de gran robustez y con la posibilidad de controlar la composición y su estructura (11). Esta metodología de trabajo permite controlar las propiedades macroscópicas del composite y se convierte en una sugerente propuesta para la búsqueda de nuevos materiales (10). Algunas de las sugerencias más interesantes que aporte mejoras en sus propiedades ópticas se están desarrollando mediante sistemas hidrofílicos-hidrofóbicos en la preparación de estructuras hexagonales altamente ordenadas y con un preciso control en el tamaño del poro obtenido (12).

En este trabajo, se describe la síntesis y caracterización de un nuevo polímero precursor poli-(9,9-bis(6'-bromohexilo)fluorenofenileno) que contiene unidades alternantes repetitivas de fluoreno/fenilideno obtenidas mediante una acoplamiento de tipo Suzuki. Esta estrategia supone una vía de preparación de polímeros conjugados solubles en agua con posibilidad de cambiar la funcionalidad de la carga e incorporarlos en estructuras ordenadas mediante soluciones homogneas. La incorporación de estos polímeros en materiales transparentes delgados se investiga por espectroscopia simple para caracterizar estos nuevos materiales potenciales en aplicaciones de sensores químicos y/o biológicos a escala nanométrica.

\section{PARTE EXPERIMENTAL}

\subsection{Materiales}

Se utilizaron los siguientes reactivos: 2,7-Dibromofluoreno, 1,6-dibromohexano, ácido 1,4-fenildiborónico,bromuro de tetrabutilamonio (TBAB), carbonato potásico, tetraetóxido de silicio (TEOS) y catalizador de Pd (II): $\mathrm{PdCl}_{2}(\mathrm{dpph})$, el copolímero de bloque Pluronic 123 de BASF y 2,7-Dibromo-9,9-bis(6'-bromohexilo)-fluoreno que se obtiene mediante catálisis de transferencia de fase, de acuerdo con procedimiento ya descrito (13).

\subsection{Instrumentación}

Los espectros de RMN de ${ }^{1} \mathrm{H}$ y ${ }^{13} \mathrm{C}$ se realizaron en un espectrómetro Varian $400 \mathrm{Mz}$, usando disolventes deuterados y los desplazamien- 
tos químicos se expresaron en partes por millón. Los espectros de FTIR se registraron con un espectrofotómetro JascoFT/IR430 en muestras preparadas sobre bromuro potásico. La cromatografía por permeación de gel (GPC) se realizó en un equipo de Waters 590, inyector manual volumen de $100 \mu \mathrm{L}$, equipado con una columna de exclusión de tamaño y un detector de índice de refracción Perkin Elmer LC-25, usando poliestireno como estándares y tetrahidrofurano (THF) como disolvente. Las muestras de polímeros se prepararon por disolución de $10 \mathrm{mg}$ de polímero en $1 \mathrm{~mL}$ de eluyente. Las muestras se pasaron a través de filtro de membrana antes de la inyección. El análisis termogravimétrico se realizó en un analizador Mettler STARe usando 7 mg de cada polímero. Los espectros UV-visible y de fluorescencia en disolución se realizaron en disolventes de grado espectroscópico, usando los espectrofotómetros Shimadzu UV-2401PC y Photon Technology International modelo C-60 (Quantum Master), respectivamente. Los espectros en fase sólida se realizaron en incidencia normal a la película, mientras que los de fluorescencia se registraron a $45^{\circ}$ respecto de la luz incidente, recalibrando la posición hasta alcanzar la intensidad máxima. Todas las muestras se midieron en las misma condiciones experimentales: rendijas, posicionamiento y temperatura.

\subsection{Síntesis de polímeros.}

\subsubsection{POLI-(9,9-BIS(6'-BROMOHEXILO)FLUORENOFENILENO) 2.}

En un matraz de $100 \mathrm{~mL}$ se añaden 2,7-dibromo-9,9-bis(6'-bromohexilo)-fluoreno $(1,958 \mathrm{~g}, 3,01 \mathrm{mmol})$, ácido 1,4-fenilborónico $(0,496 \mathrm{~g}$, $2,97 \mathrm{mmol}), \mathrm{PdCl}_{2}$ (dppf) (41 mg, 0,056mmol) y carbonato potásico $(4,534 \mathrm{~g}, 32,8 \mathrm{mmol})$. A continuación, se adicionan volúmenes equivalentes de agua $(15 \mathrm{~mL})$ y THF $(15 \mathrm{~mL})$, y se desgasifica. La mezcla se calienta a $80^{\circ} \mathrm{C}$ en agitación durante 48 horas. El residuo se precipita en metanol, se filtra y se lava con agua y acetona abundante. El polímero se purifica por disolución de cloroformo y se re-precipita en metanol varias veces. Se obtiene $1,05 \mathrm{~g}(62 \%)$ de un sólido blanco grisáceo. La caracterización estructural del polímero 2 se basa en los siguientes resultados:

${ }^{1} \mathrm{H}$ NMR $\left(\mathrm{CDCl}_{3^{\prime}} \mathrm{ppm}\right): \delta 7.84$ (br, m, 5H), 7.73-7.61 (m, 4H), 7.50 (br, m, 1H), $3.31\left(\mathrm{t}, \mathrm{J}=7 \mathrm{~Hz}, 4 \mathrm{H}, 2 \mathrm{x}-\mathrm{CH}_{2}-\mathrm{Br}\right), 2.13\left(\mathrm{br}, \mathrm{m}, 4 \mathrm{H}, 2 \mathrm{x}-\mathrm{CH}_{2}-\right.$ $\left.\mathrm{CH}_{2}-\mathrm{Br}\right), 1.71$ (br, m, 4H, 2x-CH $\left.-\mathrm{CH}_{2} \mathrm{CH}_{2}-\mathrm{Br}\right), 1.27,1.15(2 \mathrm{br}, \mathrm{m}, 8 \mathrm{H})$ and 0.81 (br, $\mathrm{m}, 4 \mathrm{H}$, Fluorene- $\left.\mathrm{CH}_{2}-\right) .{ }^{13} \mathrm{C} \mathrm{NMR}\left(\mathrm{CDCl}_{3^{\prime}} \mathrm{ppm}\right): \delta 151.94$, $140.93,140.7,140.2,128.1,126.6,121.8,120.8,55.7,40.9,34.5,33.2,29.6$, 28.3, 24.2. IR (KBr disk, $\left.\mathrm{cm}^{-1}\right): 3435,3025,2925,2845,1460,1250,890$, 810, 740, 640 and 560. GPC (THF), Mw: $8125 \mathrm{~mol} / \mathrm{g}$, Mn: $5750 \mathrm{~mol} / \mathrm{g}$, PDI: 1.41 . UV-VIS (THF): $\lambda_{\text {max }}=370 \mathrm{~nm}, \varepsilon_{\max }=6.16 \times 10^{4} \mathrm{M}^{-1} \mathrm{~cm}^{-1}$ por unidad. PL (THF, $\lambda$ ext $=370 \mathrm{~nm}$ ), $\lambda$ emission $\max =406 \mathrm{~nm}$.

\subsubsection{BROMURO DE POLI-(9,9-BIS(6'-(N,N,N- TRIMETILAMONIO)HEXILO)-FLUORENOFENILENO) 3}

A una disolución de 2 en $10 \mathrm{~mL}$ de THF (2,5\% en peso) a $-78^{\circ} \mathrm{C}$ se adicionan $2 \mathrm{~mL}$ de trimetilamina y se mantiene en agitación media hora. A continuación, la disolución se lleva a temperatura ambiente. El precipitado se re-disuelve en $10 \mathrm{~mL}$ de agua. Entonces, se adiciona $2 \mathrm{~mL}$ de trimetilamina extra. Después de 24 horas, se añade éter etílico y se extrae con agua. La capa acuosa se concentra y se lava con THF (190mg, 63\%). La caracterización estructural del polímero 3 se basa en los siguientes resultados:

${ }^{1} \mathrm{H}$ NMR (DMSO-d $\mathrm{d}^{\prime}$ ppm): $\delta$ 7.93-7.80 (br, m, 9H), 7.6-7.4 (br, m, $1 \mathrm{H}), 3.21$ (br t, $\left.\left.4 \mathrm{H}, 2 \mathrm{x}-\mathrm{CH}_{2}-\mathrm{N}^{+} \mathrm{R}_{3}\right), 2.98\left(\mathrm{~s}, 18 \mathrm{H}, 2 \mathrm{x}-\mathrm{N}^{+} \mathrm{CH}_{3}\right)_{3^{\prime}} 18 \mathrm{H}\right), 2.20$ (br, m, 4H, 2x-CH $-\mathrm{CH}_{2}-\mathrm{N}^{+} \mathrm{R}_{3}$ ), 1.49 (br, m, 4H, 2x- $\mathrm{CH}_{2}-\mathrm{CH}_{2} \mathrm{CH}_{2}-\mathrm{Br}$ ),
$1.09(2 \mathrm{br}, \mathrm{m}, 8 \mathrm{H})$ and $0.8-0.6\left(\mathrm{br}, \mathrm{m}, 4 \mathrm{H}\right.$, Fluorene- $\left.\mathrm{CH}_{2}-\right) .{ }^{13} \mathrm{C}$ NMR (DMSO-d ${ }_{6^{\prime}}$ ppm): $\delta$ 149.7, 138.1, 137.5, 136.9, 125.7, 124.1, 119.2, 118.2, 63.4, 53.4, 50.3, 46.9, 27.1, 23.7, 21.6, 20.3. IR (KBr disk, $\left.\mathrm{cm}^{-1}\right)$ : 3430, $302502925,2855,1655,1460,1255,960,902,815,740,690$ and 570. UVvis (Water): $\lambda_{\text {max }}=388 \mathrm{~nm}, \varepsilon_{\max }=2.1 \times 10^{4} \mathrm{M}^{-1} \mathrm{~cm}^{-1}$ por unidad. PL (Water, $\lambda$ ext $=385 \mathrm{~nm}), \lambda$ emission $\max =419 \mathrm{~nm}(13)$.

\subsection{Preparación de las películas.}

La incorporación de los polímeros en matrices de sílice mesoporosa en forma de películas se realiza por inmersión de acuerdo a un procedimiento descrito previamente $(12,14)$. La composición molar de la disolución acuosa es de $6 \mathrm{H}_{2} \mathrm{O}: 8,7 \mathrm{EtOH}: 0,001 \mathrm{HCl} 1 \mathrm{TEOS}$ : 0,009 P123. La disolución se agita durante media hora y se adicionan 10mg de polímero fluorescente agitando durante 3 horas. Cantidades superiores de polímero producen la precipitación en la mezcla preparada para depositar las películas. Después de la deposición, todas las muestras se curan durante 12 horas a $80^{\circ} \mathrm{C}$.

\section{RESULTADOS Y DISCUSIÓN.}

\subsection{Síntesis y caracterización de los polímeros.}

El polímero neutro poli-(9,9-bis(6'-bromohexilo)fluorenofenileno) 2, se ha sintetizado mediante un acoplamiento Suzuki usando el ácido 1,4-diborónico y 2,7-dibromo-9,9-bis(6'-bromohexilo)fluoreno 1 como precursores (Esquema 1). Sorprendentemente la reacción es muy limpia, ya que es la primera vez que se prueba este acoplamiento en presencia de sustituyentes bromoalquilo y bromoarilo y donde no se observan reacciones secundarias (eliminación o acoplamiento cruzado). El interés de esta reacción esta condicionado por varios factores:

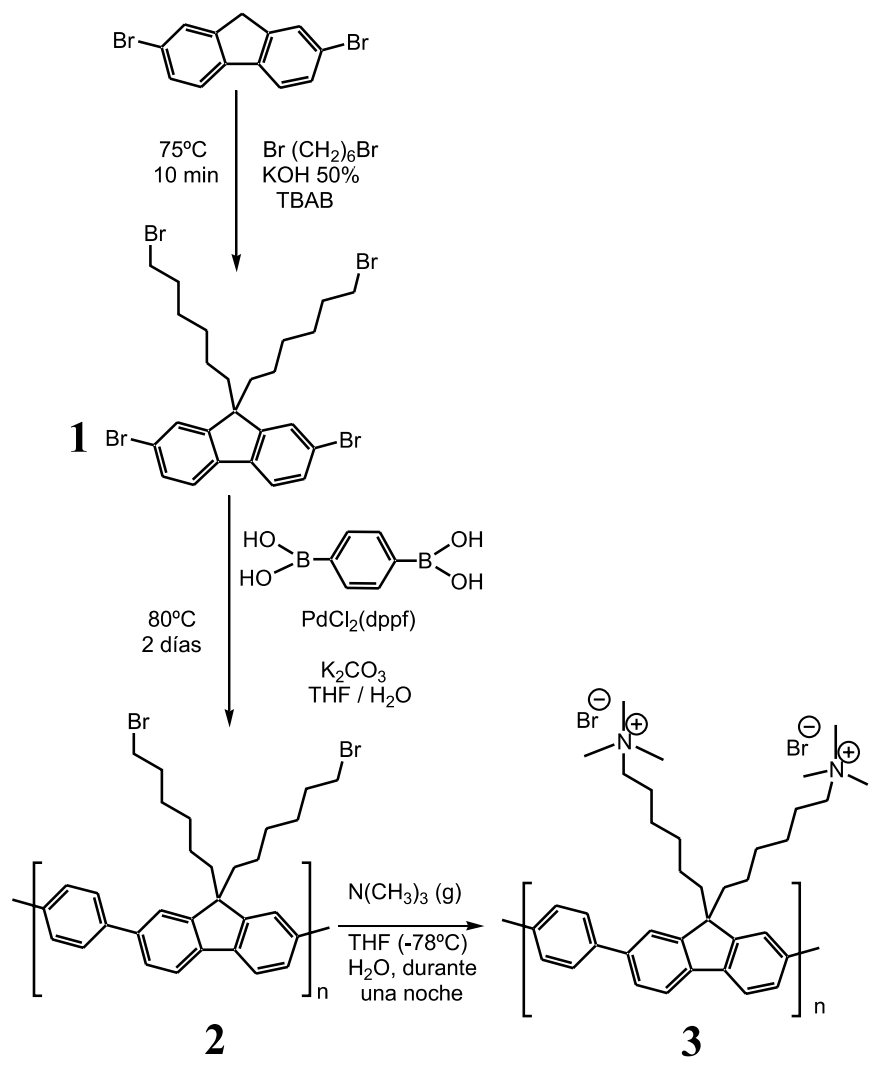

Esquema 1. Ruta sintética para obtener los copolímeros 2 y 3. 
La sencilla purificación del monómero 1, la evidente transformación mediante sustitución nucleófila en el polímero precursor 2 y que el producto final mantiene la mima distribución de pesos moleculares. El copolímero 2 se transforma en un polímero soluble en agua por tratamiento con exceso de gas de trimeltilamina (reacción Menschutkin) por la que el bromuro de alquilo se convierte en una sal de amonio. Todos los factores de la reacción facilitan el curso de la cuaternización.

Los espectros de RMN de ${ }^{1} \mathrm{H}$ y ${ }^{13} \mathrm{C}$ de los polímeros 2 y 3 son similares a los espectros analizados de polifluorenos sintetizados por este método y corresponden con las asignaciones químicas indicadas (parte experimental). El polímero 2 preparado por catálisis con Pd II en medio básico, de un fluoreno modificado 1 y un derivado diborano presenta un polímero regioregular y bien definido (se observan los seis picos aromáticos en el espectro de carbono). El desplazamiento de un pico con multiplicidad triplete centrado a 3,31 ppm se asigna al metileno adyacente al bromo terminal en el polímero 2, la desaparición de este pico en el espectro de protón de 3 es la primera evidencia de cuaternización, junto con la aparición de señales de carbono específicas en las sales amonio. La identidad del polímero 3 se ratifica por la desaparición de la banda IR de tensión de carbono-bromo a $638 \mathrm{~cm}^{-1} \mathrm{y}$ la integral relativa de la zona de los metilenos evidencian el alto grado de cuaternización existente en el polímero final 3. El peso molecular del polímero neutro 2 se caracteriza por GPC empleando calibración de poliestireno estándar, en THF como eluyente. Los resultados dan un peso molecular promedio de $8125 \mathrm{~g} / \mathrm{mol}$ y una polidispersidad de 1,4. La estabilidad térmica de ambos polímeros se analiza mediante termogravimetría en atmósfera de nitrógeno, los termogramas se muestran en la Figura 1.

En el polímero 2, la temperatura a la cual pierde el 5\% en peso es $303^{\circ} \mathrm{C}$. A partir de este valor y hasta $370^{\circ} \mathrm{C}$ sufre una pérdida de masa rápida que indica la ruptura de parte de la estructura principal. La mitad de la masa se mantiene hasta alcanzar la temperatura final del ensayo. Para el polímero 3, la primera transición comienza hacia $120^{\circ} \mathrm{C}$ con una pérdida del $10 \%$ en peso, probablemente asociado a la naturaleza higroscópica del polímero y de pérdida de bromuro de metilo (15). La segunda transición parece transcurrir a una velocidad más lenta, como se aprecia en la Figura 1, y que corresponde de nuevo con la ruptura de la cadena principal. El residuo obtenido es del $43 \%$ a $600^{\circ} \mathrm{C}$. El polímero 2 es soluble en cloroformo, diclorometano, tetrahidrofurano, tolueno, benceno y xilenos. Por otra parte, el polímero 3 es insoluble en los anteriores disolventes orgánicos y parcialmente soluble en agua, dimetilformamida y dimetilsulfóxido. El aumento de la solubilidad del polímero 3 se obtiene mediante mezclas de disolventes polares; este hecho mejora sustancialmente la solubilidad del polímero en agua.

\subsection{Propiedades ópticas.}

\subsubsection{EN DISOLUCIÓN.}

Los espectros de absorción electrónica de los polímeros de 2,7fluorenofenilideno se realizaron a temperatura ambiente. En la Figura 2 se muestra los espectros de absorción del polímero 2 en THF y del polímero 3 en disolución acuosa. Ambos espectros presentan una banda máxima a 368 y $388 \mathrm{~nm}$, respectivamente y no se aprecia absorción hacia 430nm. Los espectros de emisión de la fluorescencia de los polímeros 2 y 3 presentan una banda máxima a 406 y $419 \mathrm{~nm}$ otra banda neta a 429 y $444 \mathrm{~nm}$, respectivamente (ver Figura 2). Además, en ambos polímeros se aprecia un hombro entorno a $470 \mathrm{~nm}$, este valor depende de la polaridad del medio. Los cambios en la polaridad del disolvente inducen un cambio en la longitud efectiva de conjugación.

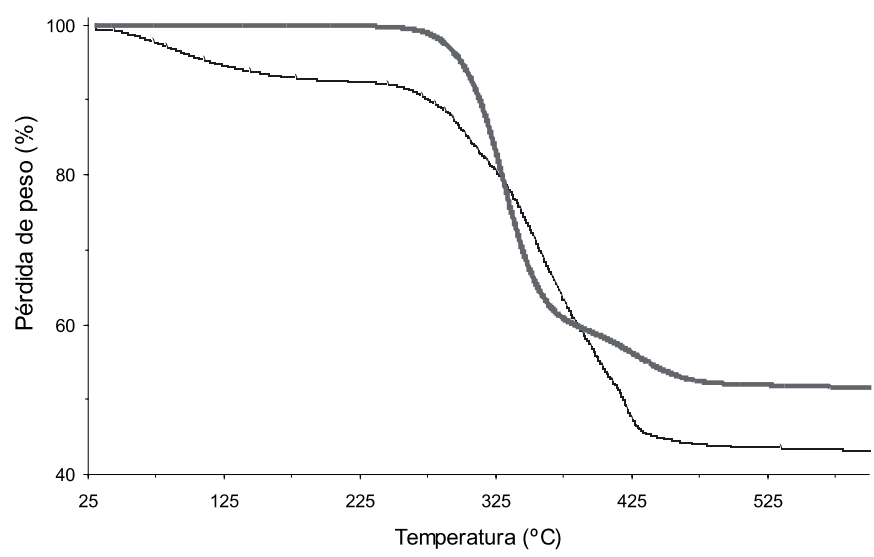

Figura 1. Termogramas del polímero neutro 2 (línea gruesa) y polifluoreno catiónico 3 (línea delgada) a $10^{\circ} \mathrm{C} / \mathrm{min}$.

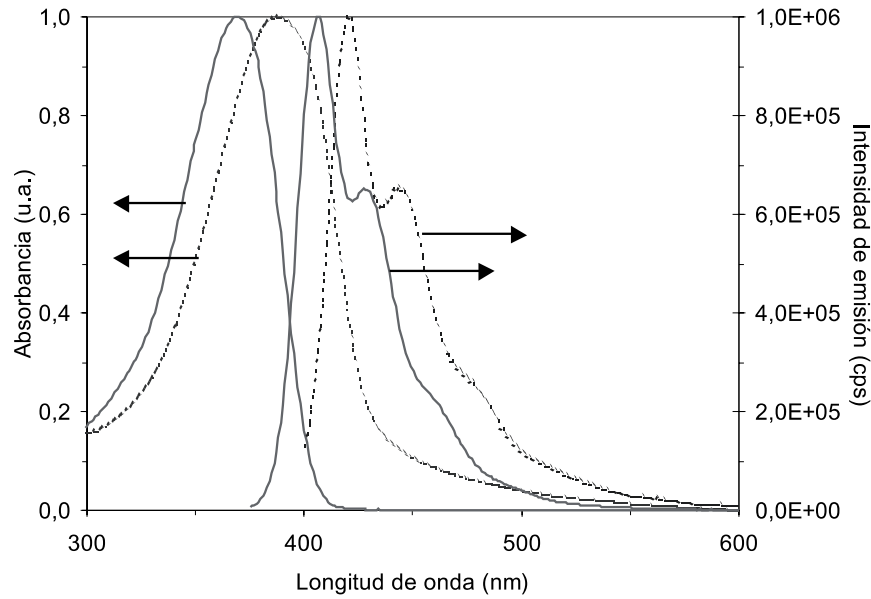

Figura 2. Espectros normalizados de absorción y fluorescencia de polifluoreno neutro 2 en THF (línea continua) y de la sal de amonio del polifluoreno 3 en agua deionizada (línea discontinua).

En nuestro caso, un ligero cambio batocrómico se observa en la banda $\pi-\pi^{*}$ entre el polímero 2 y 3 , probablemente debido a un descenso en el ángulo de torsión (anillo fluoreno y fenilo) causado por la repulsión entre cargas de los finales de la cadena alquílica, lo que motiva la estabilidad en el confórmero final de 3 .

Se realizaron ensayos de estabilidad térmica a 25 y $45^{\circ} \mathrm{C}$ de disoluciones de polímero 2 y 3 , en medios aireados, tanto en espectrocospia de absorción como de fluorescencia de emisión. En ninguno de los ensayos cinéticos registrados durante una hora se presentan variaciones significativas que pudiera generar la desactivación de los estados excitados por el oxígeno. Sin embargo, los ensayos deben realizarse con cuidado y en agitación para evitar la continua exposición de la zona excitada (fotoblanqueo) y la creación de artefactos por generación de burbujas (dispersión).

\subsubsection{EN PELÍCULAS.}

Las películas de sílice mesoestructuradas se prepararon por el procedimiento sol-gel, empleando como molde un copolímero de bloque Pluronic 123 (ver sección experimental). La incorporación directa del polímero 2 en una matriz inorgánica no fue posible debido a la precipitación del polímero bromado en la disolución preparada para la deposición por inmersión. La disolución que contiene el polímero 3 preparada para la deposición por inmersión es posible debido a la 
solubilidad del polímero iónico en agua y etanol. Se prepararon dos composiciones distintas de disolución homogénea pre-inmersión en ausencia o presencia de polímero tribloque P123, que induce en la película un orden establecido (12). En ambas composiciones se emplea la misma concentración de polímero fluorescente 3, mostrado en la Figura 3. La concentración estimada del polímero iónico en las películas se realiza por absorción espectrofotométrica y es del orden micromolar, por unidad monomérica, para un espesor de la muestra de $500 \mathrm{~nm}$ en un área de un centímetro cuadrado.

La diferencia de $5 \mathrm{~nm}$ entre ambos máximos es la observación más destacada del espectro de emisión de ambas composiciones. Si bien el espectro de emisión del polímero 3 en la película sin orden ( $\sin$ P123) mantiene el mismo desplazamiento en el máximo de absorción respecto del máximo de absorción en disolución. El empleo de un molde como el polímero tribloque P123 supone un claro desplazamiento hacia el azul. El orden inducido por el surfactante en la matriz inorgánica ha sido corroborado por difracción de rayos X (no mostrado). El motivo de este desplazamiento se puede deber al efecto del orden global conseguido en la película o bien que sea debido al cambio de entorno del polímero en el interior de un poro que produce restricciones en el movimiento de las cadenas respecto del polímero en disolución o sin orden. Independiente, este resultado es indicativo de que la movilidad de las cadenas en la película se preserva debido al orden inducido.

\section{CONCLUSIONES}

Se ha sintetizado y caracterizado un nuevo polímero bromado 2 que aporta interés en la preparación de otros polímeros conjugados cargados. Basándonos en esta ruta sintética, se ha preparado una sal de amonio cuaternaria. A partir de esta metodología se procurarán nuevas estructuras químicas de polímeros controlados. Los polímeros fluorescentes solubles en agua se han incorporado en una matriz inorgánica de sílice mesoporosa ordenada que fueron preparadas como materiales receptores. La incorporación de estos materiales orgánicos en las matrices inorgánicas potencian las posibilidades de aplicación y nuevos estudios sobre el efecto de estos nuevos materiales híbridos.

\section{AGRADECIMIENTOS}

Los autores agradecen a B. Gaylord y R.C. Hayward la colaboración prestada en la caracterización de los polímeros y a la Dra. R. Mateo por sus interesantes opiniones. Este trabajo ha sido financiado por la beca OTAN del Ministerio de Asuntos Exteriores y por el Ministerio de Ciencia y Tecnología (Proyecto MAT-2002-03515).

\section{BIBLIOGRAFÍA}

1. A. Kraft, A. C. Grimsdale, and A. B. Holmes."Electroluminescent conjugated polymers - Seeing polymers in a new light". Angew. Chem.-Int. Edit. 37 [4] 402-428 (1998).

2. Q. B. Pei and Y. Yang."Efficient photoluminescence and electroluminescence from a soluble polyfluorene". J. Am. Chem. Soc. 118 [31] 7416-7417 (1996).

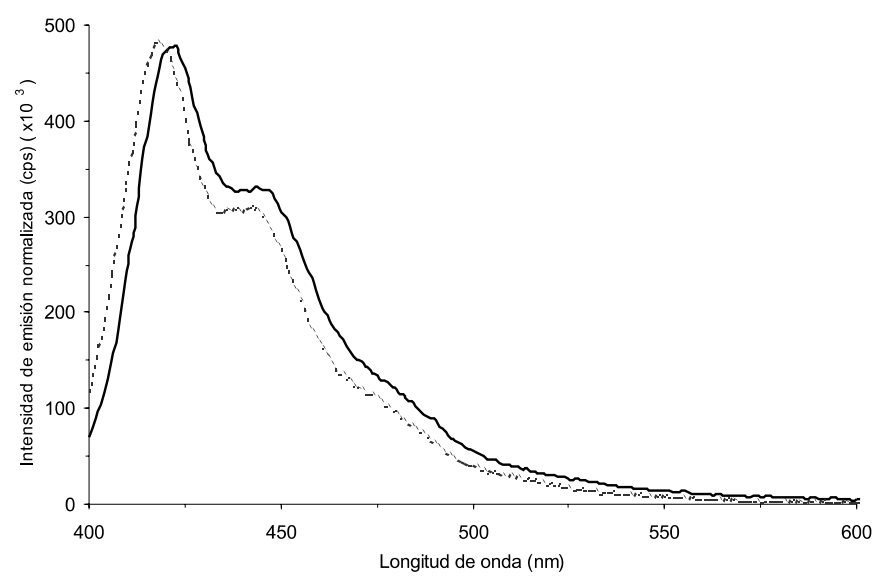

Figura 3. Espectro normalizado de fluorescencia del polímero 3 a la misma concentración en película delgada de sílice (recubrimiento). La línea continua se corresponde con una película compuesta por $\mathrm{TEOS} / \mathrm{EtOH} /$ agua/HCl/Polímero 3 y la línea discontinua con una película de TEOS/EtOH/agua/HCl/P123/Polímero 3.

3. J. S. Kim, R. H. Friend, and F. Cacialli."Improved operational stability of polyfluorene-based organic light-emitting diodes with plasma-treated indium-tin-oxide anodes". Appl. Phys. Lett. 74 [21] 3084-3086 (1999).

4. A. Charas, N. Barbagallo, J. Morgado, and L. Alcacer."Synthesis and optical properties of poly(fluorene)-based alternating copolymers". Synth. Met. 122 [1] 23-25 (2001).

5. J. Shin, D. Choi, and D. M. Shin."The characteristics of the monomer fluorene derivative in electroluminescent devices". Mol. Cryst. Liquid Cryst. 370 17-22 (2001)

6. M. T. Bernius, M. Inbasekaran, J. O'Brien, and W. S. Wu."Progress with lightemitting polymers". Adv. Mater. 12 [23] 1737-1750 (2000).

7. D. L. Wang, X. Gong, P. S. Heeger, F. Rininsland, G. C. Bazan, and A. J. Heeger."Biosensors from conjugated polyelectrolyte complexes". Proc. Natl. Acad. Sci. U. S. A. 99 [1] 49-53 (2002).

8. B. S. Gaylord, A. J. Heeger, and G. C. Bazan."DNA detection using watersoluble conjugated polymers and peptide nucleic acid probes". Proc. Natl. Acad. Sci. U. S. A. 99 [17] 10954-10957 (2002).

9. S. H. Tolbert, A. Firouzi, G. D. Stucky, and B. F. Chmelka."Magnetic field alignment of ordered silicate-surfactant composites and mesoporous silica". Science 278 [5336] 264-268 (1997).

10. J. J. Wu, A. F. Gross, and S. H. Tolbert."Host-guest chemistry using an oriented mesoporous host: Alignment and isolation of a semiconducting polymer in the nanopores of are ordered silica matrix". J. Phys. Chem. B 103 [13] 23742384 (1999).

11. R. C. Hayward, P. Alberius-Henning, B. F. Chmelka, and G. D. Stucky."The current role of mesostructures in composite materials and device fabrication". Microporous Mesoporous Mat. 44 619-624 (2001).

12. D. Zhao, P. Yang, N. Melosh, J. Feng, B. F. Chmelka, and G. D Stucky."Continuous mesoporous silica films with highly ordered large pore structures". Adv. Mater. 10 [16] 1380-1385 (1998).

13. M. Stork, B. S. Gaylord, A. J. Heeger, and G. C. Bazan."Energy transfer in mixtures of water-soluble oligomers: Effect of charge, aggregation, and surfactant complexation". Adv. Mater. 14 [5] 361-366 (2002).

14. S. Henning and L. Svensson."Production of Silica Aerogel". Phys. Scr. 23 [4] 697-702 (1981).

15. P. B. Balanda, M. B. Ramey, and J. R. Reynolds."Water-soluble and blue luminescent cationic polyelectrolytes based on poly(p-phenylene)". Macromolecules 32 [12] 3970-3978 (1999).

Recibido: 1.2.03

Aceptado: 30.11 .03 